\title{
Molecular Screening for Potential Pathogenic Organisms in Some Six Major Representative Sources of Water in Saki
}

\author{
Adeoti Olatunde Micheal", Akinbo Kehinde Oluwabusayo, Adesina David Ademola, \\ Olufemi Samson Olutope \\ Department of Science Laboratory Technology (Microbiology Option), The Oke Ogun Polytechnic Saki, Saki, Oyo State, Nigeria
}

Email address:

txy23m@yahoo.com (A. O. Micheal)

${ }^{*}$ Corresponding author

\section{To cite this article:}

Adeoti Olatunde Micheal, Akinbo Kehinde Oluwabusayo, Adesina David Ademola, Olufemi Samson Olutope. Molecular Screening for Potential Pathogenic Organisms in Some Six Major Representative Sources of Water in Saki. Frontiers in Environmental Microbiology. Vol. 6, No. 1, 2020, pp. 5-10. doi: 10.11648/j.fem.20200601.12

Received: December 23, 2020; Accepted: January 16, 2020; Published: April 8, 2020

\begin{abstract}
Water security and safety is of vital concern in Saki and the rest of the world. The aim of this was to screen major sources of water in Saki of potential pathogens of public health importance. Water samples of six different water sources were purposely collected aseptically from six different locations in Saki (well water, river water, rain water, borehole water, sachet water and bottled water); these were subjected to standard laboratory analysis. Four highly populated plates were sent for molecular characterization for Polymerase Chain Reaction and species sequencing for molecular identification. The highest total colony count was $70.0 \times 10^{-5}$ from river water followed by rain water with the lowest of $0.1 \times 10^{5} \mathrm{CFU}$ from borehole water. Molecular analysis identified amplification of isolates genes at 15000 base pair for river water and well water samples. The analysis showed that Klebsiella pneumonia (MN208186) and Enterobacter kobeii (KM593928) were the organisms found in rain water and river water respectively. The resulting nucleotide sequences was blasted at NCBI for pairwise comparison with existing data in the GenBank and their similarity was noted. The pathogenicity of the two isolates have been well-documented, making some of the water sources tested so unfit for human usage. Large-scale treatment of water sources are recommended before consumption viz-a-vis hygienic practices around water bodies.
\end{abstract}

Keywords: Fecal Coliform, Aseptically, Blasted, Enterobacter Kobeii, Water-borne Diseases, Well Water

\section{Introduction}

Many infectious diseases are associated with faecally contaminated water and are a major cause of morbidity and mortality worldwide $[1,2]$. Waterborne diseases are caused by enteric pathogens such as bacteria, viruses and parasites that are transmitted by the faecal oral route $[3,4]$. Waterborne spread of infection by these pathogenic microorganisms depends on several factors such as: the survival of these microorganisms in the water environment, the infectious dose of the microorganisms required to cause a disease in susceptible individuals, the microbiological and physico-chemical quality of the water, the presence or absence of water treatment and the season of the year $[3,5]$.

Many infectious diseases are associated with faecally contaminated water and are a major cause of morbidity and mortality worldwide $[5,6]$. Waterborne diseases are caused by enteric pathogens such as bacteria, viruses and parasites that are transmitted by the faecal oral route [7, 8]. Waterborne spread of infection by these pathogenic microorganisms depends on several factors such as: the survival of these microorganisms in the water environment, the infectious dose of the microorganisms required to cause a disease in susceptible individuals, the microbiological and physico-chemical quality of the water, the presence or absence of water treatment and the season of the year $[3,4]$.

Water is essential for the survival of every form of life and the need for water is constantly increasing due to high rates of population growth and urbanization. However, the increased demands on water for drinking, domestic agricultural and industrial purposes are not commensurate with water availability thus, posing significant risks in maintaining acceptable water quality [6]. 
Water is of course, absolutely essential to life, not only life but all life, animal and plants. Most of the biochemical reaction that occur in metabolism and growth of living cells involved water and all takes places in water [9].

Most of our water supplies are from surface water which includes rivers, streams, lakes, oceans and seas and these water bodies are likely to be polluted with domestic and industrial as well as agricultural waste. As populations increase the problem become more serious and as such water can endanger the health and life of human beings because when polluted by fecal materials it becomes potential carriers of pathogenic organisms [10].

Man uses water not only for drinking purposes but also for bathing, washing, laundering, heating, air conditioner, agriculture, stock raising and gardens. Industrial process and cooling water power and stream power, fire protection, fishing, swimming and wild life propagation and navigation. Natural water contains not only natural floral but also microorganism from soil and possible for animals or sewage. Surface waters in streams or pools and stored water in lakes and large ponds vary considerably in microbial content. Water is broadly divided into three types viz surfaces water includes: streams, rivers, lakes, seas and oceans [11].

Most bacteria are mostly commonly found ordinarily in fresh water which: Pseudomonas, Archaebacter and Vibrio. Also present are the gram positive bacterial which are found in water include: Micrococcus and Archaebacterial and actinomycetes. Tap water as one of the water sources is mostly used domestically, it was observed that tap change sometimes the tap water will be clear, this would calls for load in order to be sure if its portability [12].

The underground water, well, borehole, many people have defined the underground water in different ways: underground water is non saturated that occurs where all pore in the soil or rock counting materials are saturated [7].

Bacterial contamination of drinking water is a major public health problem worldwide, because this water can be an important vehicle of diarrhea diseases, thus, the needs to evaluate the micro-biology quality (Bacterial contamination of drinking water is a major public health problem worldwide, because this water can be an important vehicle of diarrhea diseases, thus, the needs to evaluate the microbiology quality [13]. Monitoring the micro-biology quality of drinking water is done through laboratories testing for the coliform group. The term total coliforms refers to a large group of Gram negative, rod-shaped bacterial that share several characteristics. The group includes thermo-tolerant coliforms which are of fecal origin, found in intestines of humans and warm-blooded animals, as well as the environmental species that are naturally found on plants, soil, and water. The thermo-tolerant coliforms are widely used as micro-biological parameters indicating fecal contamination while total coliform serves as a parameter to provide basic information on water quality [8]. The need assess the quality of water from some of these alternatives sources is imperatives because they have direct effects on the health of individuals. Molecular techniques are major tools for the analysis of microorganisms from food and other biological substances. The techniques provide ways to screen for a broad range of agents in a single test [14].

Rapid progress in the field of molecular biology has enabled the development of new methods for the identification of microorganisms. These techniques have proven to be particularly useful in the identification of microorganisms that are difficult to detect by phenotypic methods. Molecular biology methods can be used to confirm the presence of a specific microorganism in the tested material, as well as screen research. The most commonly used molecular methods include amplification of speciesspecific PCR products [9], PCR-based methods, and 16S rRNA/16S ribosomal DNA (rDNA) gene sequencing (currently considered the gold standard in identifying microorganism. The PCR technique was developed by [6] in the early 1980s. PCR is based on the amplification of DNA chains by repeatedly heating and cooling the sample in the presence of a polymerase and primers (i.e., short DNA fragments complementary to the sequence of the researched gene). Molecular biology methods are also used for the rapid identification of pathogens directly from clinical samples. Used PCR-based assays for the identification of Mycobacterium tuberculosis (MTB) and non-tuberculous mycobacteria (NTM). For this purpose they carried out the 16S rRNA gene sequencing by using 283 and 264 primers for PCR amplification and the Mbakt-14 primer for sequencing. Molecular-based detection gave concordant results for $97.7 \%$ and $97 \%$ of the specimens for MTB and NTM, respectively. PCR-based techniques also allowed for the explicit distinction of closely related bacteria, such as distinguishing Bacillus anthracis from other bacteria of the B. cereus group, a distinction that is not possible by any other method [15] developed a new multiplex real-time PCR assay to uniquely identify B. anthracis - a life-threatening pathogen.[16] also developed a rapid pyrosequencing assay to distinguish $B$. anthracis from the B. cereus group.

\section{Methods}

\subsection{Study Area}

The study was collected in Saki, Saki town Oyo State, western Nigeria. It lies near the sources of the Ofiki river (the chief tributary of the Ogun river), about 40 miles $(60 \mathrm{~km})$ from the Benin border. For the purpose of this study, the sampling locations were distributed over the four administrative units of Challenge, Wewe and Oke-Ogun as 1, 2 and 3 respectively.

The water sources were then categorized as ground waters sources, surface water sources and chlorinated water sources. Purpose samples or each water sources were drawn from the three administrative units.

\subsubsection{Collection of Water Samples}

During the collection of well water, tie a chord to a sterile bottle, lower it gently and collect the water sample, for river water remove the stopper then deep the sterile bottle to a 
depth of about $10 \mathrm{ml}$ directly opposes the current to collect the sample furthermore to collect pipe borne water sample, sterilize specimen bottle, crystal sterilize tap with alcohol and allow water to fairly run for five minute then aseptically collect the water with the bottle and cork up, then bottled water and sachet water were purchase in the campus and lastly for Rain water, remove the stopper then collect the sample directly and cork up.

\subsubsection{Materials Used}

Syringe was used for collection of water samples, petri dishes was used for the culturing of the microorganisms, conical flasks was used for melting and storing the media, cotton wool was used for mopping up liquid, cleaning slides, and sterilizing the working bench of the laboratory, autoclave was used for sterilizing materials like culture media, conical flasks, incubator was used for incubating at different temperature, spirit lamp was used for sterilizing the atmosphere during the work processing, test tube was used for serial dilution, test tube rack was used for putting test tube rack, Weighing balance was used for weighing and lastly measuring cylinder was used for measuring liquid substance e. g water

\subsection{Sterilization of Materials}

Working benches was sterilize with methylated and cotton wool. All the material used like flask, petri dishes, measuring cylinder were washed thoroughly and allowed to dry and there after sterilize in oven at $180^{\circ} \mathrm{C}$ for one hour all the media were also sterilized in an autoclave at $120 \mathrm{c}$ for 15 mintes.

\subsubsection{Preparation of Culture Media}

The media used were prepared according to the manufacturer's specification. These media used include nutrient agar, MacConkey agar, eosin methylene agar and Salmonella Shigella agar.

\subsubsection{Nutrient Agar}

$5.6 \mathrm{~g}$ of nutrient agar was prepared to dissolve in $200 \mathrm{ml}$ of distilled water. The mixture was heated gently to dissolve the medium completely and then sterilized in an autoclave at $121^{\circ} \mathrm{C}$ for 15 minutes.

\subsubsection{MacConkey Agar}

$11 \mathrm{~g}$ of MacConkey agar was prepared to dissolve in $200 \mathrm{ml}$ of distilled water. The mixtures was heated gently to dissolve the medium completely and then sterilized in an autoclave at $121^{\circ} \mathrm{C}$ for 15 minutes.

\subsubsection{Eosin Methylene Blue Agar}

$7.2 \mathrm{~g}$ of MacConkey agar was prepared to dissolve in $200 \mathrm{ml}$ of distilled water. The mixtures was heated gently to dissolve the medium completely and then sterilized in an autoclave at $121^{\circ} \mathrm{C}$ for 15 minutes.

\subsubsection{Salmonella Shigella Agar}

$12.6 \mathrm{~g}$ of Salmonella-Shigella agar was prepared to dissolve in $200 \mathrm{ml}$ of distilled water. The mixtures was heated gently to dissolve the medium completely and then sterilized in an autoclave at $121^{\circ} \mathrm{C}$ for 15 minutes.

\subsubsection{Isolation of Bacterial Species from Sources of Water}

One gram $(1 \mathrm{~g})$ of each water samples was homogenized in $9 \mathrm{ml}$ of sterile distilled water and vortexed for 10 minutes, then serial dilution of water were prepared. One millimeter $(1.0 \mathrm{ml})$ each of $10^{3}, 10^{5}$ dilution were inoculated on sterile nutrient agar plates and MacConkey plate using pour plate method; the plates were incubated at $28+2^{\circ} \mathrm{C}$ before averted. The number of colonies in the surface of the plate were counted and expressed. Furthermore, counting and picking of colonies from plates $10^{-3}$ and $10^{-5}$ for the organism to survive. Pure culture of the bacteria where obtained by sub culturing on nutrient agar, MacConkey agar, eosin methylene blue agar and Salmonella-Shigella agar respectively.

\subsection{Characterization of Bacterial Isolates}

The selected bacteria were characterized by obtaining their colony morphologies like shape and gram staining.

\subsubsection{Gram Staining Techniques}

A small drop of distilled water was placed in a clean slide. The inoculating wire loop was sterilized by flaming it until it was red hot. The loop was allowed to cool and picked a small portion of the isolate and smeared in the drop of water on the slide. A thin smear was made and allow it to air dried after this, this smear was stained with $1 \%$ crystal violet for 60 seconds and washed with tap water, it was stained with gram iodine for 60 second and washed with water. It was then decolorized with $75 \%$ alcohol for 30 seconds and rinsed with tap water. The slide was counter stained with safranin for 30 seconds and washed off with tap water and air dried. The slides were observed under the microscope under oil immersion objective lens $(\times 100)$. Gram negative appeared red while gram positive appear purple [15].

\subsubsection{Biochemical Test}

Several biochemical test were conducted for the pure isolates which are: A loopful of the isolate was placed on glass slide, a drop of 3\% of hydrogen peroxides (H2O2) was added to the isolates, so production of gas bubbles of foaming indicated positive result while no bubbles show a negative result.

\subsection{Molecular Identification (PCR) Techniques}

\subsubsection{Electrophoresis for DNA and PCR}

Measure $1 \mathrm{~g}$ of agarose (for DNA); $2 \mathrm{~g}$ of agarose for PCR, Mix agarose powder with $100 \mathrm{~mL}$ 1xTAE in a microwavable flask and Microwave for 1-3 min until the agarose is completely dissolved (but do not over boil the solution, as some of the buffer will evaporate and thus alter the final percentage of agarose in the gel then wait till agarose solution cool down to about $50^{\circ} \mathrm{C}$ (about when you can comfortably keep your hand on the flask), about 5 mins. Then add $10 \mu \mathrm{L}$ EZ vision DNA stain. EZ vision binds to the DNA and allows you to visualize the DNA under ultraviolet 
(UV) light. Pour the agarose into a gel tray with the well comb in place and Place newly poured gel at $4{ }^{\circ} \mathrm{C}$ for $10-15$ mins OR let sit at room temperature for 20-30 mins, until it has completely solidified.

\subsubsection{Loading Samples and Running an Agarose Gel}

Add loading buffer to each of your DNA samples or PCR products once it is solidified, place the agarose gel into the gel box (electrophoresis unit). Then Fill gel box with 1xTAE (or TBE) until the gel is covered. Carefully load a molecular weight ladder into the first lane of the gel and load your samples into the additional wells of the gel to run the gel at 80-150 V for about 1-1.5 hours then turn OFF power, disconnect the electrodes from the power source, and then carefully remove the gel from the gel box to visualize DNA fragments or PCR product under UV trans illuminator.

\subsubsection{PCR Mix Components}

The PCR mix is made up of $12.5 \mu \mathrm{L}$ of Taq $2 \mathrm{X}$ Master Mix from New England Biolabs (M0270); $1 \mu \mathrm{L}$ each of $10 \mu \mathrm{M}$ forward and reverse primer; $2 \mu \mathrm{L}$ of DNA template and then made up with $8.5 \mu \mathrm{L}$ Nuclease free water.

\section{Result}

\section{DNA}

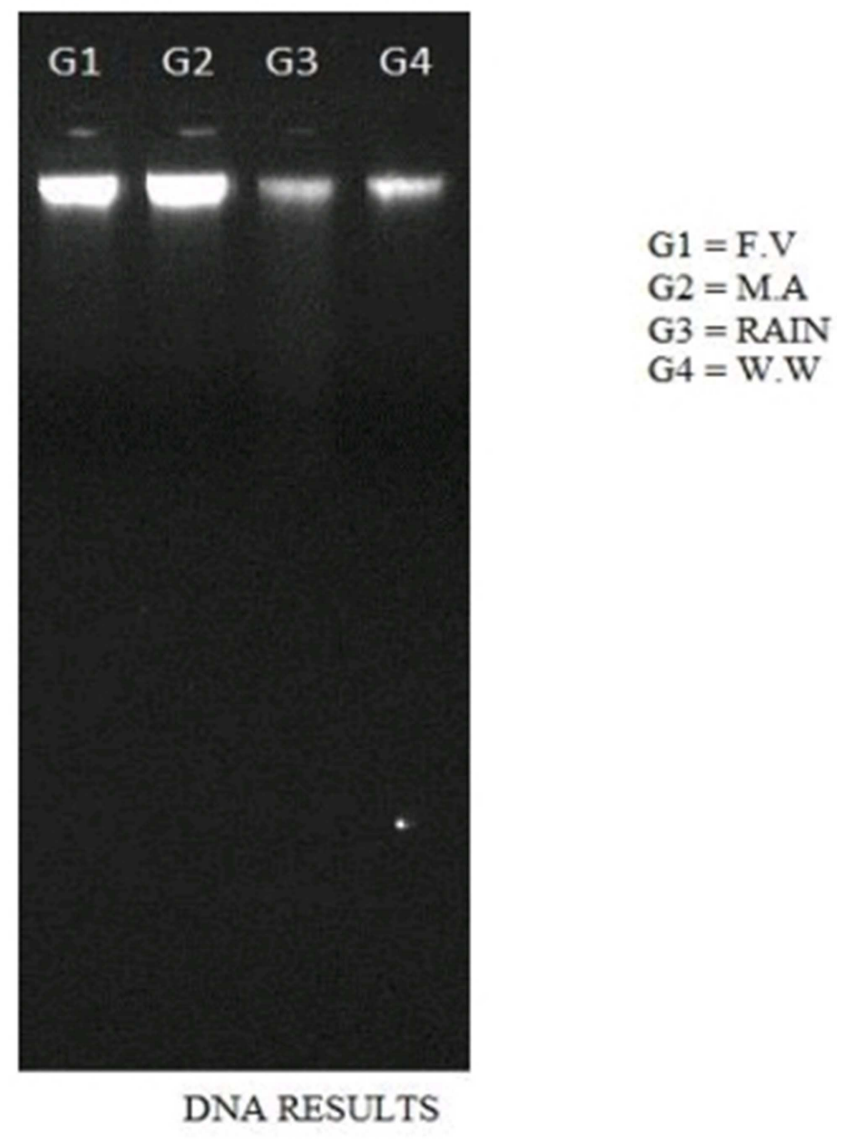

Figure 1. Bacterial DNA samples (G3, G4) loaded into the wells showing amplification

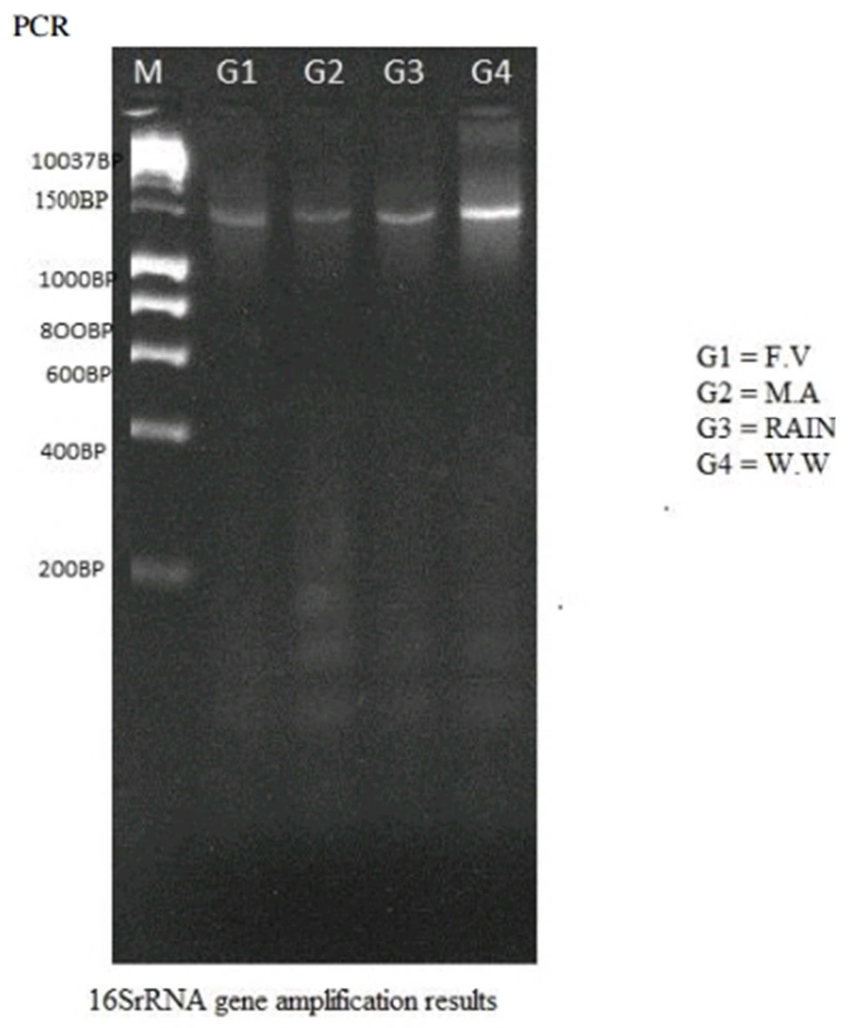

Figure 2. $1.0 \%$ Agarose gel electrophoresis showing molecular weight of bacteria DNA samples.

Sample Code: Rain

Identified Organism: Enterobacter kobeii strain Sdv1B10 \% PAIRWISE IDENTITY: 72.95\% NCBI ACCESSION NUMBER: KM593928 E Value: 0 Isolate Sequences:

GGGGTTAATCTATGGGATCATGGCTCAGGTGCGCTGG GAACCACCCCTTTAAAGTTT

GACGGCCTCAGGGGGGCTGGATGTCCCCTAAAAATT GTGGGGGGGGGGTATCTCCT

GTGAACACTATATATAACCCCGTATCGCCTCACAACA

CAAAAGAGGGGCCTTTTCGC

CTCTCGTGCTCTCATATGTCCCCATATGGTTATATAT

AATGTGGGGTGAAGGCTCTCC

CCTGCGACAATATCCATCTGTGCTCTGAGAAGAACC CCCCCCACTGTGGCTGTGACA

CACTCCCCACACCCCCAGGGGACGCCACTGTGGAA

AATTGTGCACTGTGGGCACAC

CTGTTACGCCCCTGTGCCGCGTGTAAAAAAAGCCTT

CTCGTTGTGAAATATTTTTTCC

GCGGAGAAAAGCGTTTTGATTATTATCCTCTCCGATA GTGACTTCCCCCACAAAAAA

CACCCGCTATATCTCTGCGCCCGCCCCCGCGATTATA CGGGGGGCGCGCGTGTTTCT

CAGATTTAGTGCGCATAACGCACACGCGCGCTCTGT

GTAGTCTCATATGAAATCCCC

CCGGGTCTCCCCGTGAAATCTGTTTTTAAACTGTGAC GCTATAGTCTCGTGTAGAGG

GGGGATAATTTCTCCGTGTGTCGCGGAAATGCGCATA

GATCTGGGAGGAAAAATCA

CCGGGTGGGAAAGCGGCCCCCCCTGGGGAAAAACA 
GTAGACTCTCGATGTGCAAAT

CGCGTGGGAGCACAAAACGAGTATATACCCCTGTGT

ATTTCTTCCACCCCTATACAA

CGATCTCCCATGTGAGATGTTTGCGCCCTTTGGACGC

GGGGTTTTCCGAGAGTATAA

CGGTTTTATATCTTACCCCCCCTCGTGGGAGAACAGC

CCCCCCACGAGTTTTTAACTC

CTCAAATGATATTTGTGCGACGGGGGCGCCACCCAC

AAGAAGCGTGTGTGACACTC

GATGGAGTGTTATATATCTGCAGACGCGCACCGTGAG

AAAATACCTCTATCACAAAT

CCTGTGGAAATTCACAGAAGAGTTTCTCT

Sample Code: Well Water Identified Organism: Klebsiella pneumoniae strain

201-b \% PAIRWISE IDENTITY: $87.56 \%$ NCBI ACCESSION NUMBER: MN208186 E Value: 0 Isolate Sequences:

GGGGTATCATATGTCGATCATGGCTCAGGTGCGGTGG

GATCACCCCCTAAAAGTTTG

ACGGCCTCGGGGGGGTGGGAACCCCCTTAAAATTGT TGGAGGGGGATAACTACTGG

AAACAGTATCTAATACAGCATAACGCCGCACGACCC

AAGTGGGGGACCTTCTCGCC

TCTTGCGATCTCATGTGCCCCGATGGGATTAGCTATTA

GGTGGGGTAACGGCTCACC

TATGCGACAATCCCTATCTGGTCTGAGAGAATGACCC CCCCCACTGGAACTGAGACA

CGGTCCACACTCCCACGGGAGGCGCCACTGGGGAAT

ATTGCACAATGTGCGCAAGC

CCGATGCACCCCTGCCGCGTGTGTGAAGAAAGCCTT

CTGGTTGTAAAGCACTTTCTC

CGCGGGAGGGAGGCGATAAGGTTAATATCCTTGTCG

ATTGTCGTTACCCGCACAAA

AAACACCGGCTATATCTGTGCGACCACCCGCGGTAAT

ACGGGGGGTGCGAGCGTGA

ATCTGAATTACTGGGGCGTAAAACGCACACGGGCGG

TCTGTGAAGACGGATGTGAA

ATCCCCCGGGCTCTCCCCTGGGAACTCCGTTCTAAA

CTGGGGACGCTATAGTCTCTG

TAGAGGGGGGGAATAATTCCCCGGTGTGTCGCTGGA

AATGCGCAATAGATATGGGA

GGAAAAACCCCGGTGGGCGAAAGGCGGCCCCCCCC

TGGGACAAAAAACTCACGGC

CTCTCGATGCGAAAAACGGCGGGGGCAGCAAAAAA

AACGAATATATAATACCCCTG

GGTTCTTCCCCCCGCCCGGTAAAACAGAATGATTC

GTACTTTTGTGAGGGTTTGTGG

CTCCCCTTTATGAGACGGTGGGTCTCTCCGGAAACAT

ATACACCCGTGTATAATCCC

ACCCCCGCCGCCTGCGGGAAGTACAGCGCCCCGCG

ACGAGTCTAAAACCTCCCAAA

ATGTATATATTGTGTACGGCGGGGGCTCCCCCCACAC

CAGACGGGCTTGCTGCAGAC

AGCGGTTGGGTTTTATATATTACTCTGACCGCACGCG

CGGAAAAATACTTTATCATG

CGTGGTGCTCTGTGCGCACATC

\section{Discussion}

The analysis examination carried out on the water sources that serves for private and public water supply were intended to assist in the determination of the quality of drinking water in independence layout of Saki town. World Health Organization $[17,18]$ have stipulated standards for water meant for human consumption and the result of the present investigation did not meet the standards except the tap water. The total bacterial count of the water sources showed general variation from different samples. The result shows that River water sources has most poor quality in terms of contamination with coliforms. The borehole water sources showed that there are few content of coliform likewise bottle water unlike other water sources [19]. The Isolation of coliform in other sources of water is an indication of feacal contamination of the water sources like the Well, River water, Rain water and bottle water [21]. Water is one of the most important vehicles for the transmission of infectious diseases and fecal contamination of water sources particularly in developing and underdeveloped countries has been welldocumented.

\section{Conclusion}

River water, well water, Rain water and bottled water has been found to be unsafe for human consumption and for industrial uses because of large number of bacterial that grew on Agar plate incubated for 24 hours except being properly treated before consumption to avoid outbreak of water transmitting diseases. Disinfection such as boiling, chlorination, using ultraviolet rays or ozonation is recommended before consumption and use of water for drinking and domestic purpose. This would minimize health risk associated with other chemical consumption in the form of firewood boiling water as well. Surface, ground and chlorination urban water sources in Saki were contaminated with bacteria and cysts to levels regarded as unsafe as per the standards for portable water. This makes the water unsafe for direct drinking and use in food processing.

\section{References}

[1] Julian, T. R., MacDonald, L. H., GUO, Y., Marks, S. J., Kosek, M., Yori, P. P., Pinedo, S. R., Schwab, K. J. (2013). Fecal indicator bacterial contamination of fomites and Household demand for surface disinfection products: A case study from peru. The American journal of tropical medicine and hygiene 89: 869-872.

[2] Lay, B., \& Miter b. (2010). Emtrcolities epidemic as a result of contamination of drinking Water through E. colisterotype abstract $2 b$ Pp 42.

[3] Diosi, A. L., and mitard. (2011). E. coli type soil and OSS in drinking Water abstract $7 \mathrm{~b} 1$.

[4] Dondero, W. C. (2009) Advanced Applied Microbiology Vol. 3 Academic press, New York London. Pp 77-178. 
[5] Gaecdrich, E. E. (2012). Waterborne pathogen in water pollution microbiology R. Mitchell, John wiley and sons inc. New York Pp 102.

[6] Gopinath, A., pratapChandran, R., Vysakhi, MV., Anu, A., (2006). Physical and bacteriological quality Of well water samples from kanakkaypanchayath, kottayam district, Kerala State, India International Journal of Plant and Animal environ 2012.

[7] Pelezer, M. J., R. G. D \& R. D. (2012). Microbiology (3 ${ }^{\text {rd }}$ edition) M. C Grawhill book company Pp 143-767.

[8] Shibata, T. Solo-Gabriele, H. M. Fleming, BLE, Elmir S. (2004) Monitoring marine recreational water quality using multiple microbial indicators in a urban tropical environment. Water Res 38: 3119-31.

[9] Callaway, A., Kostrzewa, M., Willershausen, B., Schmidt, F., Thiede, B., Kupper, H., \&Kneist, S. (2013). Clin. Lab. 2013. 121225 .

[10] Carpenter beth, V. J. (2007). Indicator of recreational water quality American Society for Testing and Mathials.

[11] Kelman, A., Person, L. H., and Herbert, T. T. (2007). Plant disease conference report $\mathrm{Pp} 802$.

[12] Bonde, S. J. (2009). Advances in agnatic microbiology vol. 1 London and New York Academic press.

[13] Suthar, S. Chhimpa, V. Singh, S. (2009) Bacterial contamination in drinking water: A case study in rural areas of Northern Rajasthan, India. Environ Monitoring \& Assessment 159: 43-50.
[14] Field, D. Wills, C. (1998). Abundant microsatellite polymorphism in saccharomyces in eight prokaryotes and S. Cerevisiae, result from strong mutation pressures and variety of selective force Process. National Acadamic of. Science U. S. A. 95 : $1647-1652$.

[15] WHO. (2011). Guideline for drinking water quality $3^{\text {rd }}$ edition. World Health Organization.

[16] Ahmod, N. Z., Gupta, R. S., \& Shah, H. N. (2011). J. M microbiology. Methods 87, 278-285 doi: 10. /j. mimet 2011. 08.015 .

[17] WHO, U. (2000). Global water supply and sanitation assessment 2000 report. Geneva: World Health Organization.

[18] WHO, U. (2014) Progress in drinking water and Sanitation: 2014 update. World.

[19] Nuria, M. C., Rosyid, A., Sumantri, S. (2009). UjikandunganbakteriEscherichia colipada air minumisiulangdari depot air miniumisiulang di kabupatenrembang testing of Escherichia coli bacteria content drinking water refill from drinking water refill depot in Rembang sub-district. MEDIAGRO 5.

[20] Boria, A. \&Mitarb [2009]. Inter-countries epidemic as a result of contamination of drinking water with E. coli strain 0124 . Abstract 2bi. Pp. 178-180.

[21] Kahinda, J. M., A. E Taigbenua, E. S. B Lilieb and Boroto, R. J. (2009). GIS- based decision Support system for rain water harvesting (RHAESS). Physics and Chemistry of the Earth parts. 